ARTICLE

Received 9 Aug 2013 | Accepted 17 Dec 2013 | Published 24 Jan $2014 \quad$ DOl: 10.1038/ncomms4139

\title{
Two potential therapeutic antibodies bind to a peptide segment of membrane-bound IgE in different conformations
}

Hsing-Mao Chu' ${ }^{1}$, Jon Wright ${ }^{1,2}$, Yueh-Hsuan Chan ${ }^{3}$, Chien-Jen Lin ${ }^{1}$, Tse Wen Chang ${ }^{1} \&$ Carmay Lim²,4

IgE mediates hypersensitivity reactions responsible for most allergic diseases, which affect $20-40 \%$ of the population in developed countries. A 52-residue domain of membrane-bound IgE (mlgE) called C $\mathrm{C}_{\mathrm{m}} \mathrm{X}$ is currently a target for developing therapeutic antibodies; however, its structure is unknown. Here we show that two antibodies with therapeutic potential in IgE-mediated allergic diseases, which can cause cytolytic effects on mlgE-expressing B lymphocytes and downregulate IgE production, target different conformations of an intrinsically disordered region (IDR) in the extracellular $C_{\varepsilon m} X$ domain. We provide an important example of antibodies targeting an extracellular IDR of a receptor on the surface of intended target cells. We also provide fundamental structural characteristics unique to human mlgE, which may stimulate further studies to investigate whether other monoclonal antibodies (mAbs) targeting intrinsically disordered peptide segments or vaccine-like products targeting IDRs of a membrane protein can be developed.

\footnotetext{
${ }^{1}$ The Genomics Research Center, Academia Sinica, Taipei 115, Taiwan. ${ }^{2}$ Institute of Biomedical Sciences, Academia Sinica, Taipei 115, Taiwan. ${ }^{3}$ Fountain Biopharma Inc. Taipei, Taipei 115, Taiwan. ${ }^{4}$ Department of Chemistry, National Tsing Hua University, Hsinchu 300, Taiwan. Correspondence and requests for materials should be addressed to T.W.C. (email: twchang@gate.sinica.edu.tw) or to C.L. (email: carmay@gate.sinica.edu.tw).
} 
O malizumab is a humanized $\mathrm{mAb}$ that binds to the $\mathrm{CH} 3$ domain of human IgE, near the binding site for the highaffinity type I IgE Fc receptors (FceRI). It can neutralize free IgE and inhibit the IgE-mediated allergic pathway without sensitizing mast cells and basophils ${ }^{1,2}$. Omalizumab (trade name Xolair) has been shown in 60 completed clinical trials to be efficacious and safe for treating various IgE-mediated allergic diseases such as allergic asthma, allergic rhinitis and food allergy $^{3-6}$ as well as several non-allergic diseases that involve mast cells, such as chronic idiopathic urticaria ${ }^{7,8}$. Xolair has been approved in over 90 countries for treating patients with severe allergic asthma, uncontrollable even with high corticosteroid doses. These pharmaceutical developments have validated the IgE pathway as an effective therapeutic target for treating IgE-mediated allergic diseases ${ }^{2,9-11}$.

The discovery of a 52-residue domain termed C $\varepsilon \mathrm{mX}$ as part of the predominant isoform ${ }^{12}$ of human membrane-bound $\varepsilon$ chain $(\mathrm{m} \varepsilon)$ (Fig. 1a) provides another approach to target the $\operatorname{IgE}$ pathway by reducing $\operatorname{IgE}$ production ${ }^{2,12}$. The gene segment encoding C $\varepsilon \mathrm{mX}$ is only found in the $\mathrm{m} \varepsilon$ of recently evolved primates, namely, the New-world monkeys and Old-world monkeys (including Homo sapiens) ${ }^{13}$. CemX is present in $\mathrm{m} \varepsilon$ but not in membrane-bound immunoglobulin of other isotypes. To date, only two allelic forms differing by a leucine or valine at position 16 along the CemX sequence (which generally does not affect antigenicity) have been found ${ }^{14}$. Although the threedimensional (3D) structure of CemX remains unsolved, previous work showed that two cysteines in CemX form an intrachain disulfide bond (Cys-18 with Cys-39 or Cys-41), while the other two cysteines form an interchain bridge ${ }^{15}$. CemX is thought to play a role in determining the outcome of cell signaling, following $\mathrm{B}$ cell receptor engagement ${ }^{16}$; however, its function has not been

a

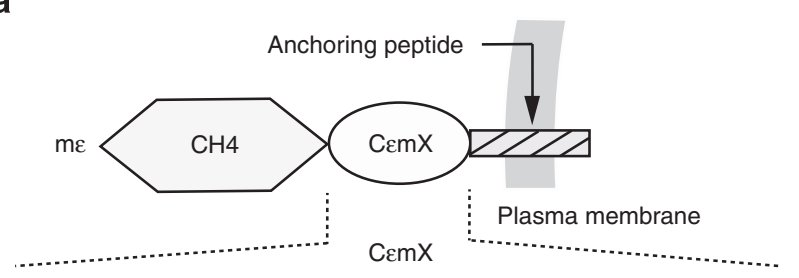

${ }^{1}$ GLAGGSAQSQRAPDRVLCHSGQQQGLPRAAGGSVPHPRCHCGAGRADWPGPP ${ }^{52}$

b

P-01 ${ }^{1}$ GLAGGSAQSQRAPDRVLC ${ }^{18}$

P-02 ${ }^{1}$ GLAGGSAQSQRAPDRVL ${ }^{17}$

P-03 ${ }^{1}$ GLAGGSAQSQRAPDR ${ }^{15}$

P-04 ${ }^{6}$ SAQSQRAPDRVLCHS ${ }^{20}$

P-05 ${ }^{6}$ SAQSQRAPDRVLC 18

P-06 $\quad{ }^{7}$ AQSQRAPDRVLC ${ }^{18}$

P-07 ${ }^{8}$ QSQRAPDRVLC ${ }^{18}$

P-08 ${ }^{9}$ SQRAPDRVLC ${ }^{18}$

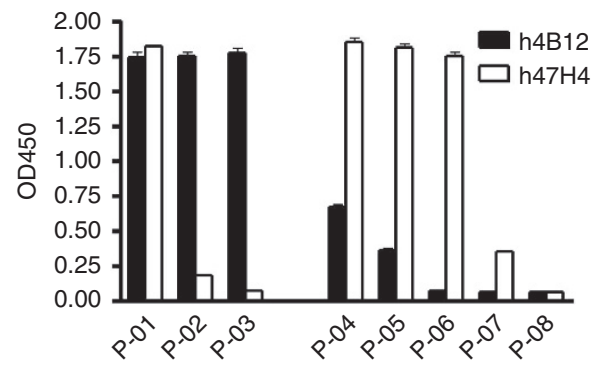

Figure 1 | Epitope mapping of anti-CemX mAbs, h4B12 and h47H4.

(a) The CemX sequence in human mlgE. (b) Reactivity of h4B12 and h47H4 with synthetic peptide segments of $\mathrm{C} \varepsilon m \mathrm{X}$ (P-01 to P-08).

The mean and s.d. of triplicate measurements are shown. fully elucidated. Unlike omalizumab that binds to both membrane-bound $\operatorname{IgE}(\mathrm{mIgE})$ and free $\operatorname{IgE}$, an anti-CemX antibody targets only $\mathrm{mIgE}$ and therefore can target $\mathrm{mIgE}$ expressing B lymphoblasts and memory B cells to downregulate IgE production ${ }^{17,18}$ without being neutralized by free IgE. Thus, a therapeutic anti-C\&mX antibody could potentially be administered less frequently at smaller doses than omalizumab.

Here we analyse binding of two potential therapeutic humanized mAbs against human CemX to their target. The antibody h4B12 from our group, derived from the parental murine mAb 4B12 (ref. 17), is in preparation to enter human clinical studies. The antibody h47H4 (quilizumab) from Genentech, derived from the parental murine mAb $47 \mathrm{H} 4$ (ref. 18), is in Phase IIb clinical trial for patients with moderate to severe allergic asthma. The $2.4-\AA$ crystal structure of the complex of the Fab of h47H4 and a 35-residue CemX peptide (PDB ID $3 \mathrm{hr} 5)^{18}$ shows that an 11 -residue segment, ${ }^{6} \mathrm{SAQSQRAPDRV}{ }^{16}$, is bound to h47H4, while the exact binding mode of h4B12 is not clear. In this work, we map the epitopes of these two potential therapeutic mAbs, determine the crystal structure of h4B12 Fab in complex with its peptide epitope, and present evidence that the dimeric CemX domain is intrinsically disordered.

\section{Results}

Epitope mapping analysis of two anti-CemX mAbs. To define the epitopes of these two humanized mAbs, various CemX peptides were synthesized and their affinities for h47H4 (which was prepared according to its published $\mathrm{V}_{\mathrm{H}}$ and $\mathrm{V}_{\mathrm{L}}$ sequences) and h4B12 were analysed using enzyme-linked immunosorbent assay (ELISA). Remarkably, the results show that h4B12 and h47H4 recognize overlapping, but different epitopes, and they do not crossreact with each other's epitope. The first $15 \mathrm{C} \varepsilon \mathrm{mX}$ residues $\left({ }^{1}\right.$ GLAGG SAQSQ RAPDR ${ }^{15}$ ) bound h4B12 but not h47H4 (Fig. 1b, P-03), whereas residues $7-18\left({ }^{7}\right.$ AQSQ RAPDR VLC $\left.{ }^{18}\right)$ bound h47H4 but not h4B12 (Fig. 1b, P-06).

The results in Fig. $1 \mathrm{~b}$ also indicate the $\mathrm{C} \varepsilon \mathrm{mX}$ residues that are critical for binding each of the two anti-CemX mAbs. For h47H4, Cys-18 appears critical as its deletion nearly abolished binding to h47H4 but did not affect binding to h4B12 (Fig. 1b, P-01 versus $\mathrm{P}-02)$. Ala-7 is also involved in discriminating the two mAbs, as its deletion dramatically reduced binding to h47H4 (Fig. 1b, P-06 versus P-07). For h4B12, on the other hand, the first five CemX residues, ${ }^{1}$ LLAGG $^{5}$, contributes to binding $\mathrm{h} 4 \mathrm{~B} 12$, as their deletion attenuates binding to h4B12 but not to h47H4 (Fig. 1b, P-01 versus $\mathrm{P}-05$ ). The next residue, Ser-6, also contributes to binding h4B12, as its deletion reduced binding to h4B12 but not to h47H4 (Fig. 1b, P-05 versus P-06). Despite these differences, the two mAbs bind to a common 10-residue segment, ${ }^{6} \mathrm{SAQSQ}$ RAPDR $^{15}$.

The crystal structure of h4B12-peptide complex. To determine how h4B12 binds to its epitope, we have determined the crystal structure of the h4B12 Fab in complex with its peptide epitope (excluding Gly-1), ${ }^{2}$ LAGG SAQSQ RAPDR ${ }^{15}$ at 1.92 - $\AA$ resolution (Fig. 2 and Table 1); Gly-1 was modeled into the 3D structure of the N-terminal CemX using the PyMOL program ${ }^{19}$ and energyminimized to allow contact analysis of h4B12 Fab with its recognized full-length epitope, ${ }^{1}$ GLAGG SAQSQ RAPDR ${ }^{15}$. The van der Waals and hydrogen-bonding interactions between h4B12 and its epitope shown in Fig. 3a appear consistent with the h4B12 ELISA results. The first five CemX residues, ${ }^{1} \mathrm{GLAGG}^{5}$, bind h4B12 mainly via non-bonded contacts with Ile-56(H2), Gly-58(H2), Gly-91(L3), Val 94(L3) and Met-95(H3). The next CemX residue, Ser-6, forms a backbone-backbone hydrogen bond with Gly-91(L3), while Gln-10 forms a sidechain-sidechain 


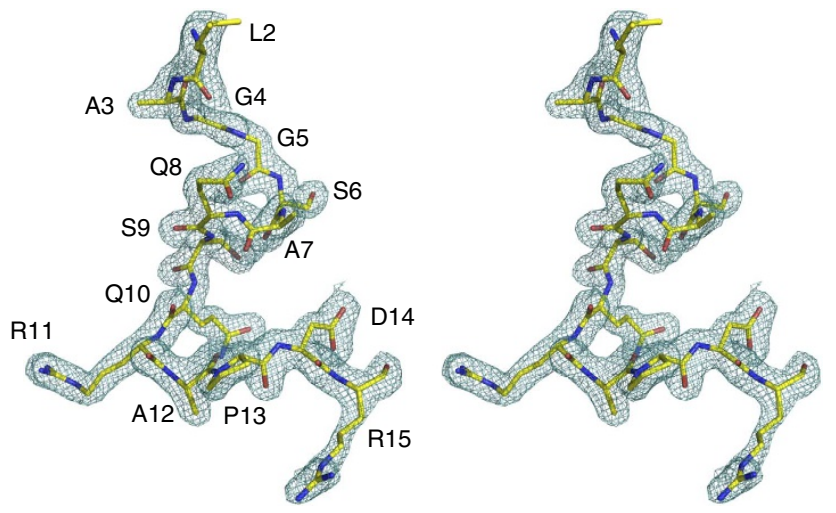

Figure 2 | The electron density map of the C€mX peptide bound by h4B12. ${ }^{2}$ LAGGSAQSQRAPDR ${ }^{15}$ peptide was clearly defined in the $2 F_{0}-F C$ electron density map contoured at $1.0 \sigma$.

hydrogen bond with Asp-32(H1). The C-terminal ${ }^{11} \mathrm{RAPDR}^{15}$ CEmX residues contact h4B12 through five hydrogen bonds with the L1, L2, H1 and H3 loops.

Conformations of the peptides bound by h4B12 and h47H4. To determine the binding-mode differences between h4B12 and $\mathrm{h} 47 \mathrm{H} 4$ to CemX, we compared the peptide complex structure of h4B12 solved herein with that of h47H4 (PDB ID: $3 \mathrm{hr} 5)^{18}$ in Fig. $3 \mathrm{~b}$. The two mAbs bound the same peptide segment ${ }^{6} \mathrm{SAQSQ}$ RAPDR $^{15}$ in significantly different conformations, as shown in Fig. 3b. When the Ala-12 and Pro-13 backbone atoms of both peptides were superimposed, the ends of the two peptides diverged, as evidenced by a huge separation of $18.7 \AA$ between the Ser- $6 \mathrm{O}^{\gamma}$ atoms and $11.3 \AA$ between the Arg-15 $\mathrm{C}^{\zeta}$ atoms. This disparity in the conformations of the two peptides bound by $\mathrm{h} 4 \mathrm{~B} 12$ and $\mathrm{h} 47 \mathrm{H} 4$ suggests that this $\mathrm{C} \varepsilon \mathrm{mX}$ region may be intrinsically disordered.

CemX domain is intrinsically disordered. To ascertain that CemX contains intrinsically disordered region (IDRs), we employed various IDR algorithms to predict disorder in the entire CemX domain ${ }^{20}$ and measured the circular dichroism spectra of dimeric CemX. Both approaches confirmed that CemX contains IDRs. All the IDR algorithms ${ }^{20}$ predict disorder for the first 12 CemX residues (Fig. 4a). The meta-meta predictor GSmetaDisorderMD2 (ref. 20), which was the top-scoring method in the CASP9 benchmark, predicted the entire CEmX domain to be disordered. Notably, CemX consists of $73 \%$ disorder-promoting residues (E, K, R, G, Q, S, P or A) and only $17 \%$ order-promoting ones (I, L, V, W, F, Y or C). In accord with the predictions, molecular dynamics simulations of the free peptides starting from their bound structures (PDB ID: 4lkx and 3hr5) show that both antigenic peptides do not adopt welldefined conformations in water (see Methods). Furthermore, the far UV circular dichroism spectra in Fig. $4 \mathrm{~b}$ shows that dimeric CemX, unlike ovalbumin, contains no signature profiles for ordered secondary structures (negative peaks at 208 and $222 \mathrm{~nm}$ for $\alpha$ helices and a negative peak at $217 \mathrm{~nm}$ for $\beta$ sheets) but exhibits a negative peak at $\sim 200 \mathrm{~nm}$ characteristic of a random coil.

\section{Discussion}

Previous studies have shown that IDR-containing proteins exist broadly in all life forms and play crucial roles in recognition, regulation, cell signaling and control pathways ${ }^{21-24}$, and IDRs are

\begin{tabular}{|c|c|}
\hline & h4B12-Fab/peptide complex \\
\hline \multicolumn{2}{|l|}{ Data collection } \\
\hline Space group & $P 2_{1} 2_{1} 2_{1}$ \\
\hline \multicolumn{2}{|l|}{ Cell dimensions } \\
\hline$a, b, c(\AA)$ & $42.0,107.1,110.2$ \\
\hline$\alpha, \beta, \gamma\left({ }^{\circ}\right)$ & $90.0,90.0,90.0$ \\
\hline Resolution $(\AA)$ & $50.0-1.92(1.99-1.92)^{\star}$ \\
\hline$R_{\text {sym }}(\%)$ & $9.0(58.7)$ \\
\hline$|/ \sigma|$ & $29.3(3.8)$ \\
\hline Completeness (\%) & $99.9(98.9)$ \\
\hline Redundancy & $7.1(6.7)$ \\
\hline \multicolumn{2}{|l|}{ Refinement $\dagger$} \\
\hline Resolution $(\AA)$ & 28.4-1.92 \\
\hline No. of reflections & 36,692 \\
\hline$R_{\text {work }} / R_{\text {free }} \ddagger(\%)$ & $17.9 / 22.6$ \\
\hline \multicolumn{2}{|l|}{ No. of atoms } \\
\hline $\mathrm{Fab}$ & 3,270 \\
\hline Peptide & 98 \\
\hline Water & 261 \\
\hline \multicolumn{2}{|l|}{ B-factors } \\
\hline Fab & 32.7 \\
\hline Peptide & 30.5 \\
\hline Water & 37.4 \\
\hline \multicolumn{2}{|l|}{ R.M.S deviations } \\
\hline Bond lengths $(\AA)$ & 0.01 \\
\hline Bond angles $\left({ }^{\circ}\right)$ & 1.4 \\
\hline \multicolumn{2}{|c|}{$\begin{array}{l}\text { R.M.S, root mean square. } \\
\text { *Highest resolution shell is shown in parentheses. } \\
\dagger \text { The quality of the refined structure was validated by using MOLPROBITY53, which show all } \\
\text { residues in the favoured and allowed regions in the Ramachandran plot. } \\
\ddagger R \text { free is the } R \text { factor calculated using } 5 \% \text { of the resolution data chosen randomly and omitted } \\
\text { from the start of refinement. }\end{array}$} \\
\hline
\end{tabular}

prevalent in disease-associated proteins such as p53, c-Myc transcription factor, $\alpha$-synuclein and glycogen synthase kinase $3 \beta^{24-26}$. Since IDRs often interact with multiple partners with high specificity but low affinity ${ }^{22,27}$, they are considered as drug targets for small molecules that interfere with the binding of these IDRs to their binding partners ${ }^{28}$. Indeed, small drug-like molecules that block the binding of an IDR in p53 to murine double minute 2 have been developed and the drug candidates such as MI-219 and Nutlin-3 are undergoing Phase I clinical trial for the treatment of retinoblastoma cancer ${ }^{29}$. In addition, several antibodies targeting disordered loop regions of diseaseassociated proteins such as viral protein hemagglutinin ${ }^{30}$ have been reported. However, no study had shown that antibody drug molecules can target an extracellular IDR of a receptor on the surface of a targeted cell type with the desired cytolytic effects.

This study shows that potential therapeutic antibodies targeting IDRs of a protein drug target may be prepared. An advantageous feature of an IDR is its ability to adopt different structures upon binding to different partners. In analogy to the C-terminal IDR of p53 that is involved in 'one-to-many' binding ${ }^{31}$, the N-terminal IDR of CemX can adopt a twistedcoil or an extended-coil conformation upon binding to h4B12 or h47H4, respectively. The fact that a region of a protein exists in an intrinsically disordered state does not preclude the generation of antibodies binding to this region with high affinity and high specificity. Antibodies generated by immunization of a peptide segment of a protein can bind to the free peptide segment with high affinity. Although some anti-peptide antibodies do crossreact with comparable affinity 
a

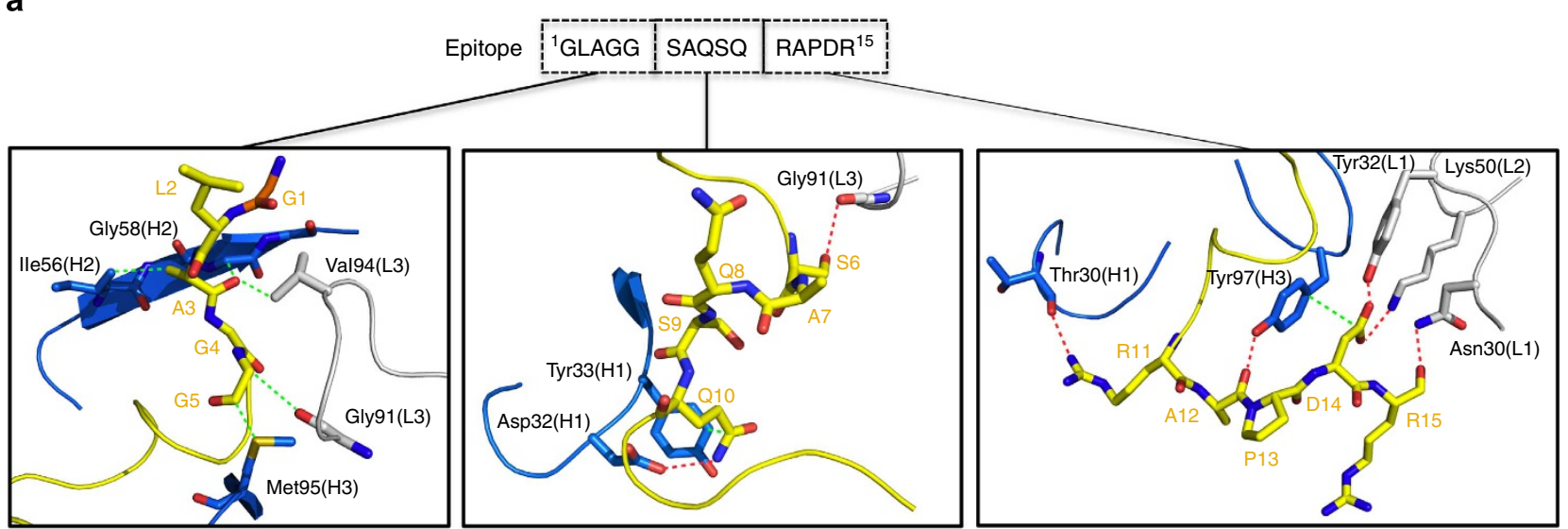

b
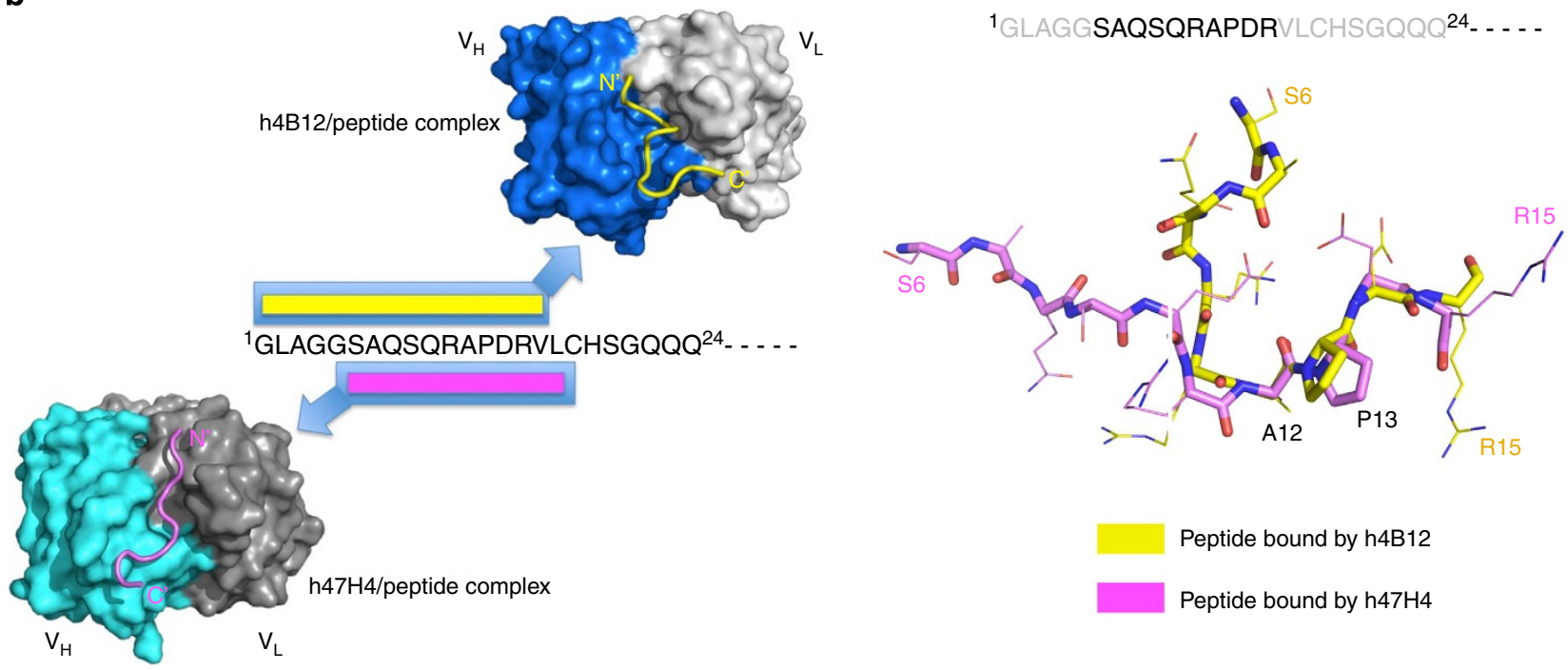

Peptide bound by h4B12

Peptide bound by h47H4

Figure 3 | The interaction between h4B12-Fab and its antigenic peptide and the conformations of the antigenic peptides when bound by h4B12 and h47H4. (a) The h4B12 Fab forms non-bonded contacts with ${ }^{1} \mathrm{GLAGG}^{5}$ (left), two hydrogen bonds with ${ }^{6} \mathrm{SAQSQ}^{10}$ (middle) and five hydrogen bonds with ${ }^{11} \mathrm{RAPDR}^{15}$ (right). The $\mathrm{C}_{\varepsilon m X}$ peptide is in yellow with modeled $\mathrm{Gly}-1$ in orange, the $h 4 B 12 V_{L}$ is in grey and its $V_{H}$ is in blue. $L$ and $H$ denote complementarity-determining regions of $V_{\mathrm{L}}$ and $\mathrm{V}_{\mathrm{H}}$, respectively. Hydrogen bonds (red dashed lines) and van der Waals contacts (green dashed lines) are defined by a donor atom to an acceptor atom distance $\leq 3.5$ and $\leq 4.0 \AA$, respectively. (b) The 1.9- $\AA$ structure of h4B12 $F_{V}$ (light grey and dark blue) and ${ }^{2}$ LAGG SAQSQ RAPDR ${ }^{15}$ (yellow) and the 2.4- $\AA$ structure of h47H4 (dark grey and light blue) and ${ }^{6}$ SAQSQ RAPDRV ${ }^{16}$ (violet) (left panel). Ala-12 and Pro-13 backbone nonhydrogen atoms in the common segment ( ${ }^{6}$ SAQSQRAPDR ${ }^{15}$ ) are superimposed to reveal the different peptide conformations in the two crystal structures (right panel).

with the native proteins when the peptide is flexible within the native structure-for example, antibodies against peptide segments of hemagglutinin ${ }^{32}$ and lysozyme ${ }^{33}$-they generally bind to the denatured protein ${ }^{34}$. In contrast, h4B12 and h47H4 bind to the same CemX peptide segment of native mIgE in quite different conformations with high affinity and specificity. Furthermore, they cause the lysis of mIgE-expressing B cells, thus downregulating IgE production ${ }^{17,18}$. Notably, h47H4 has advanced to phase IIb clinical trial.

Both h4B12 and h47H4 can bind to mIgE on B lymphocytes and mediate cytolytic mechanisms on mIgE-expressing B cells through apoptosis and antibody-dependent cellular cytotoxicity ${ }^{17,18}$. The antigenic sites for those two mAbs are not blocked by possible CemX-binding partner(s) or by associated molecules on the cell surface. This is important since not all regions of CemX are accessible to mAbs ${ }^{17}$. The IDR algorithms (Fig. 4a) predicted that the C-terminal CemX segment of about 10 residues is also intrinsically disordered. Earlier work showed that mAbs could bind strongly to this peptide segment in CemX-containing proteins in a cell-free system $^{35}$, but not to mIgE on $B$ lymphocytes. This suggests that the terminal segment of CemX is blocked by adjacent molecules on the cell surface ${ }^{17}$ or by possible CemX-binding partner(s).

This study suggests a novel way to elucidate intrinsic disorder by inducing a disordered region to adopt different structures upon binding to different mAbs and determining the mAb-bound structures using X-ray crystallography, NMR or other spectroscopic methods. It has provided the first structural characterization of the entire dimerized CemX region (Fig. 4b), which is not only an important drug target, but also is unique in that $\mathrm{C} \varepsilon \mathrm{mX}$ is found solely in mIgE but not in free IgE or other immunoglobulin classes. The significance of an intrinsically disordered CemX domain is that more than one specific, high-affinity antibody can be developed to target CemX in native mIgE and cause cytolytic mechanisms. It prompts investigations as to whether CemX or its IDRs could be used to prepare vaccine-like products for use in 
$\mathbf{a}$
1
10
20
30
40
50

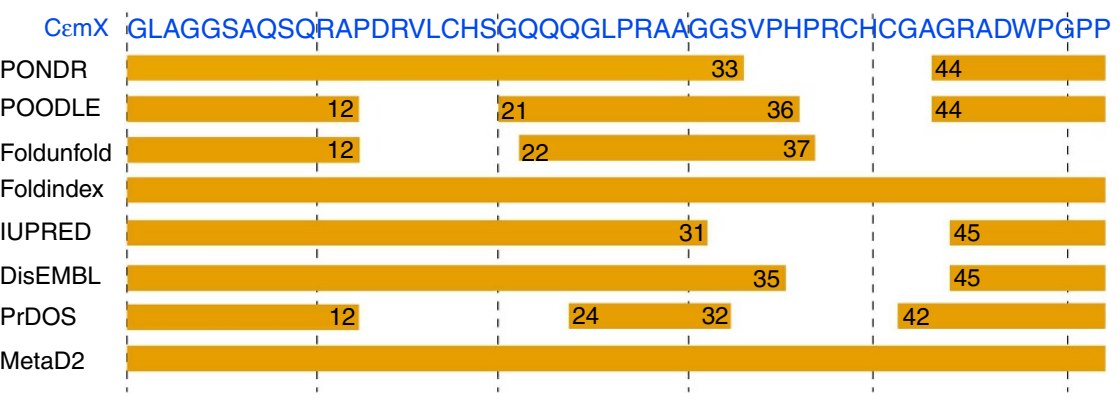

b

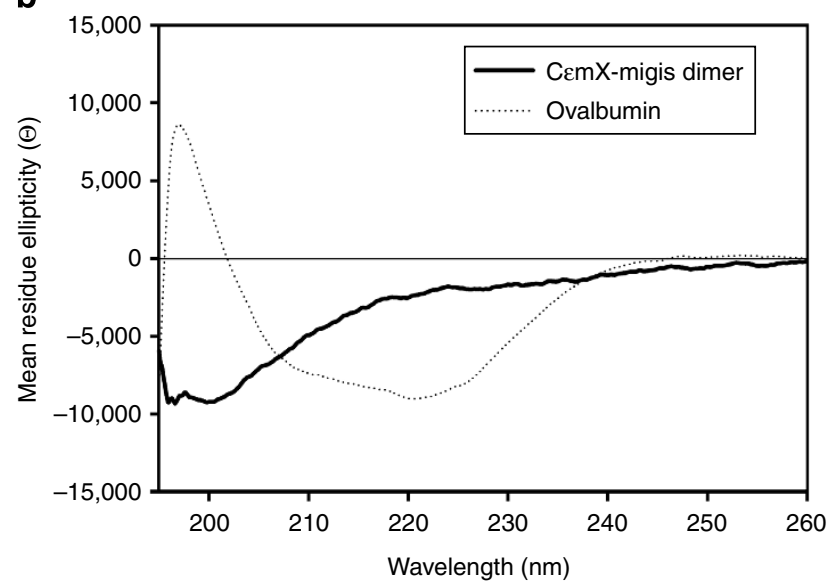

Figure 4 | Intrinsic disorder of the free $\mathbf{C} \boldsymbol{\varepsilon} \mathbf{X}$ peptide or domain. (a) Prediction of disorder (orange) in the CemX domain by available servers. (b) Far UV circular dichroism spectra of dimeric CemX and the migis- $\varepsilon$ domain (black curve) and ovalbumin (dotted grey curve).

patients to elicit antibody response against CemX and hence downregulate the mIgE-expressing $\mathrm{B}$ cells. Hence, this work should encourage the development of therapeutic antibodies that bind with high specificity and affinity to extracellular IDRs in other drug target proteins.

The findings herein highlight the difference between the two isoforms of $\mathrm{mIgE}$ found in humans. If the asymmetric sIgE structure were to apply to $\mathrm{mIgE}$ (whose structure is unknown), mIgE would require a substantial conformational change to enable the Fab regions to bind antigen ${ }^{11}$. Our results suggest that the highly flexible, disordered CemX region of mIgE enables such a conformational change, allowing the Fab regions to reorient and bind antigen. Thus, compared with the non-CemX-containing $\mathrm{mIgE}$, the additional flexibility provided by the long-form mIgE has advantages for binding antigen, possibly accounting for its dominance over its short-form counterpart ${ }^{12}$.

\section{Methods}

ELISA binding assays. Peptides used for epitope mapping were synthesized the Peptide Core Laboratory of the Genomics Research Center in Academia Sinica, Taiwan. The ultra pure peptides used for crystallization were synthesized by Genomics Inc. (Taipei, Taiwan) and dissolved in $10 \mathrm{mM}$ Tris buffer stock solution at a concentration of $30 \mathrm{mg} \mathrm{ml}^{-1}$. For epitope mapping, peptides of $10 \mu \mathrm{g} \mathrm{ml}^{-1}$ were coated on wells of a 96 -well plate at $4{ }^{\circ} \mathrm{C}$ overnight. After blocking with $1 \%$ bovine serum albumin, $1 \mu \mathrm{g} \mathrm{ml}^{-1}$ of h4B12 or h47H4 was added to the wells. The plate was then incubated at room temperature for $2 \mathrm{~h}$, and horseradish peroxidaseconjugated goat anti-human IgG diluted 1:20,000 was added to the wells. After incubating for $1 \mathrm{~h}$ at room temperature, the signal was developed by TMB solution and the absorbance at $\mathrm{OD}_{450}$ was measured.

IgG1 Fc•C\&mX + migis and h4B12 Fab expression and purification. The IgG1 Fc•CemX + migis (migis is the extracellular portion of the C-terminal transmembrane peptide segment) and h4B12 Fab proteins were expressed in FreeStyle
293F suspension culture cell expression system and medium (Invitrogen, CA). Transfection of targeted genes into $293 \mathrm{~F}$ cells was performed at a cell density of $10^{7}$ cells $\mathrm{ml}^{-1}$ in 600-ml culture in 2-L Erlenmeyer flasks using linear polyethylenimine with an average molecular weight of $25 \mathrm{kDa}$ (Polysciences, Warrington, PA) as a transfection reagent. The transfected cells were incubated at $37^{\circ} \mathrm{C}$ for $4 \mathrm{~h}$ in an orbital shaker ( 125 r.p.m.), and their cell density was then

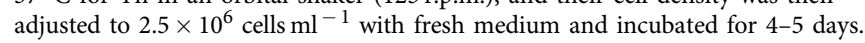
Culture supernatants were harvested, and IgG1 Fc•CemX + migis and h4B12 Fab in the media were purified using Protein A and KappaSelect chromatography, respectively.

Crystallization and X-ray structure determination. The h4B12-Fab $\left(44 \mathrm{mg} \mathrm{ml}^{-1}\right)$ and the CemX peptide ${ }^{2}$ LAGSAQSQ RAPDR ${ }^{15}\left(10 \mathrm{mg} \mathrm{ml}^{-1}\right)$ were mixed and subjected to crystallization trials using the hanging-drop vapour diffusion method with a protein-to-reservoir ratio of $1 \mu \mathrm{l}: 1 \mu \mathrm{l}$. From the initial crystallization screening, crystals of h4B12 complexed with the peptide were obtained using a reservoir containing $0.1 \mathrm{M}$ sodium acetate trihydrate at $\mathrm{pH} 4.6$, $25 \%$ PEG4000 and $0.2 \mathrm{M}$ ammonium sulfate. The crystals were grown at $16^{\circ} \mathrm{C}$ and the reservoir volume was $500 \mu \mathrm{l}$. They were soaked for $5 \mathrm{~s}$ in the reservoir solution containing $25 \%(\mathrm{v} / \mathrm{v})$ glycerol as a cryoprotectant. X-ray data were collected at a wavelength of $0.97622 \AA$ on beamline BL13C1 at NSRRC in Hsinchu, Taiwan using the MAR300 CCD detector. All data were processed and integrated using the programs Denzo and Scalepack in the HKL2000 suite ${ }^{36}$ (see Table 1). Calculation of Matthews coefficient suggested that the asymmetric unit contains one protein molecule. The h4B12-Fab structure was solved by molecular replacement with the Phaser Program ${ }^{37}$ using the light chain of the Fab structure (PDB ID: 3AAZ) and the heavy chain of the Fab structure (PDB ID: 2JIX) separately as a search model. The programs $\operatorname{Coot}^{38}$, PHENIX ${ }^{39}$ and REFMAC5 (ref. 40) were used in model building and refinement, while the sigma A-weighted $2 F_{0}-F_{c}$ electron density map guided the model-building process. In the complex structure, most of the h4B12 Fab structure was modeled into the good $2 F o-F c$ map; the electron density for residues Ser-127-Thr-131 of the heavy chain was not visible. Unambiguous electron density of Ala-3-Asp-14 of the bound peptide was seen at 2.5- $\sigma$ cutoff in the initial $F o-F c$ difference map. Among these residues, the electron density maps for the side chain of Ser-6, Gln-10 and Pro-13 were most defined; therefore, the model of the bound peptide was built using these three residues as the base. The complex structure was improved by rigid-body, positional and restrained 
refinement with individual isotropic B-factors using PHENIX ${ }^{39}$ and REFMAC5 (ref. 40). The statistics of the final model are listed in Table 1.

Molecular dynamics simulations. The 14-residue ${ }^{2}$ LAGGSAQSQRAPDR ${ }^{15}$ and 11-residue ${ }^{6}$ SAQSQRAPDRV ${ }^{16}$ peptides were extracted from their X-ray structures complexed with h4B12 (this work) and h47H4 (3h5r), respectively, and were subjected to 16 ns molecular dynamics simulations using the CHARMM ${ }^{41}$ version 37 program and the CHARMM27 all-atom parameter set ${ }^{42}$. All Asp residues were deprotonated, whereas Arg residues were protonated. The resulting peptide with a net charge of +1 was neutralized by adding a chloride counterion at the position of highest electropositivity with the constraint that the counterion was $\geq 7 \AA$ from the peptide surface. The neutral system was solvated in a truncated octahedron containing TIP3P water molecules ${ }^{43}$, resulting in a total of 15,209 or 15,191 atoms. To relieve any bad contacts in the solvated peptide structure, the water molecules were subjected to rounds of minimization with constraints on the protein heavy atoms. The resulting solvated system was subjected to molecular dynamics at a mean temperature of $300 \mathrm{~K}$ using a $2 \mathrm{fs}$ timestep, periodic boundary conditions, van der Waals interactions shifted to zero at $12 \AA$ and electrostatic interactions treated via the particle mesh Ewald summation method ${ }^{44}$. Initially, $40 \mathrm{ps}$ of dynamics was performed with the peptide backbone atoms and counterions restrained by a harmonic potential, which was then removed for the rest of the $16 \mathrm{~ns}$ simulation where the coordinates were saved every $2 \mathrm{ps}$. The saved 7,500 conformations were clustered pairwise using the Maxcluster program ${ }^{45}$, which computes the root-mean-squared deviations of the $\mathrm{C}^{\alpha}$ atoms and clusters conformations that are within $2.5 \AA$ of the cluster centroid. For the 14-residue ${ }^{2}$ LAGGSAQSQRAPDR ${ }^{15}$ peptide, $99.5 \%$ of the 7,500 saved conformations could be grouped into 47 clusters with the largest three clusters accounting for only $35 \%$ of the sampled conformations, whereas for the 11-residue ${ }^{6}$ SAQSQRAPDRV ${ }^{16}$ peptide, $99.8 \%$ of the conformations could be assigned to 17 clusters with the top three clusters accounting for $73 \%$ of the sampled conformations.

Disorder predictions. The CemX sequence was submitted to the following publicly available intrinsic disorder predictors: PONDR VL-XT ${ }^{46}$ (pondr.com/ index.html), POODLE-S ${ }^{47}$ (http://mbs.cbrc.jp/poodle/poodle-s.html), FoldUnfold ${ }^{48}$ (http://skuld.protres.ru/ mlobanov/ogu/ogu.cgi), Foldindex ${ }^{49}$ (http://bip.weizmann.ac.il/fldbin/findex), IUPRED ${ }^{50}$ (http://iupred.enzim.hu, using short algorithms), $\operatorname{PrDOS}^{51}$ (http://prdos.hgc.jp/cgi-bin/top.cgi), DisEMBL ${ }^{52}$ (http://dis.embl.de) and MetaDisorderMD2 (MetaD2) ${ }^{20}$ (http://iimcb.genesilico.pl/ metadisorder/). We used the default parameters in each algorithm and refer the reader to the cited references for details of each method.

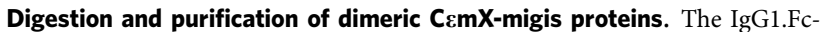
CemX-migis fusion protein was incubated with recombinant enterokinase (rEK) to remove the dimeric CemX-migis regions and nonCemX residues, Gly and Ser at the $\mathrm{N}$-terminus. The rEK and the fusion protein at a ratio of 1:50 (unit $\mu \mathrm{g}^{-1}$ ) were incubated at $25^{\circ} \mathrm{C}$ overnight. rEK was removed using EKapture Agarose (Novagen) according to the manufacturer's instructions. The mixture without rEK was applied to a Protein A column to remove the IgG1 Fc and incompletely digested material. The dimeric CemX-migis was collected in the flow-through; EKapture Agarose was again added to remove any rEK contaminant and buffer-exchanged into PBS buffer using a PD-10 column.

Circular dichroism spectra. Circular dichroism spectra were monitored on a Jasco 815 dichrograph using 1-mm-thick quartz cells in $10 \mathrm{mM}$ sodium phosphate buffer, $\mathrm{pH} 7.4$, at $20^{\circ} \mathrm{C}$. They were measured between 195 and $260 \mathrm{~nm}$, with a scanning speed of $20 \mathrm{~nm}$ min and a data pitch of $0.1 \mathrm{~nm}$. Spectra were averaged from three scans and smoothed using the 'means-movement' smoothing procedure implemented in the SpectraManager package. The contribution of buffer was subtracted from experimental spectra. Mean ellipticity values per residue $([\Theta])$ were calculated as $[\Theta]=3,300 m \Delta \mathrm{A} /(l c n)$ in degrees $\mathrm{cm}^{2} \mathrm{dmol}^{-1}$, where $m$ is the molecular mass in Daltons, $\Delta \mathrm{A}$ is differential absorbance of left and right circularly polarized light, $l$ is the path length equal to $0.1 \mathrm{~cm}, c$ is the protein concentration equal to $0.1 \mathrm{mg} \mathrm{ml}^{-1}$ and $n$ is the number of residues, which is 138 for dimeric CemX-migis.

\section{References}

1. Chang, T. W. The pharmacological basis of anti-IgE therapy. Nat. Biotechnol. 18, 157-162 (2000).

2. Chang, T. W., Wu, P. C., Hsu, C. L. \& Hung, A. F. Anti-IgE antibodies for the treatment of IgE-mediated allergic diseases. Adv. Immunol. 93, 63-119 (2007).

3. Holgate, S. T., Djukanovic, R., Casale, T. \& Bousquet, J. Anti-immunoglobulin E treatment with omalizumab in allergic diseases: an update on antiinflammatory activity and clinical efficacy. Clin. Exp. Allergy 35, 408-416 (2005).

4. Busse, W. W. et al. Randomized trial of omalizumab (anti-IgE) for asthma in inner-city children. N. Engl. J. Med. 364, 1005-1015 (2011).
5. Kopp, M. V. et al. Omalizumab (Xolair) in children with seasonal allergic rhinitis: leukotriene release as a potential in vitro parameter to monitor therapeutic effects. Pediatr. Allergy Immunol. 18, 523-527 (2007).

6. Sampson, H. A. et al. A phase II, randomized, doubleblind, parallelgroup, placebocontrolled oral food challenge trial of Xolair (omalizumab) in peanut allergy. J. Allergy Clin. Immunol. 127, 1309-1310, el301 (2011).

7. Kaplan, A. et al. Omalizumab in patients with symptomatic chronic idiopathic/ spontaneous urticaria despite standard combination therapy. J. Allergy Clin. Immunol. 132, 101-109 (2013).

8. Maurer, M. et al. Omalizumab for the treatment of chronic idiopathic or spontaneous urticaria. N. Engl. J. Med. 368, 924-935 (2013).

9. Holgate, S. et al. The anti-inflammatory effects of omalizumab confirm the central role of IgE in allergic inflammation. J. Allergy Clin. Immunol. 115, 459-465 (2005).

10. Casale, T. B. \& Stokes, J. Anti-IgE therapy: clinical utility beyond asthma J. Allergy Clin. Immunol. 123, 770-771, e771 (2009).

11. Gould, H. J. \& Sutton, B. J. IgE in allergy and asthma today. Nat. Rev. Immunol. 8, 205-217 (2008).

12. Peng, C. et al. A new isoform of human membrane-bound IgE. J. Immunol. 148, 129-136 (1992).

13. Wu, P. C. et al. The IgE gene in primates exhibits extraordinary evolutionary diversity. Immunogenetics 64, 279-287 (2012).

14. Wan, L. et al. Genetic variations in the $\mathrm{C}$ epsilon $\mathrm{mX}$ domain of human membrane-bound IgE. Immunogenetics 62, 273-280 (2010).

15. Bestagno, M. et al. Membrane immunoglobulins are stabilized by interchain disulfide bonds occurring within the extracellular membrane-proximal domain. Biochemistry 40, 10686-10692 (2001).

16. Poggianella, M., Bestagno, M. \& Burrone, O. R. The extracellular membraneproximal domain of human membrane IgE controls apoptotic signaling of the B cell receptor in the mature B cell line A20. J. Immunol. 177, 3597-3605 (2006).

17. Chen, J. B. et al. Unique epitopes on CemX in IgE-B cell receptors are potentially applicable for targeting IgE-committed B cells. J. Immunol. 184, 1748-1756 (2010).

18. Brightbill, H. D. et al. Antibodies specific for a segment of human membrane IgE deplete IgE-producing B cells in humanized mice. J. Clin. Invest. 120, 2218-2229 (2010).

19. DeLano, W. L. The PyMol molecular graphics system. (Version 1.5 Schrodinger, LLC, 2008). URL: http://www.pymol.org/.

20. Kozlowski, L. P. \& Bujnicki, J. M. MetaDisorder: a meta-server for the prediction of intrinsic disorder in proteins. BMC Bioinformatics 13, 111 (2012).

21. Dyson, H. J. \& Wright, P. E. Intrinsically unstructured proteins and their functions. Nat. Rev. Mol. Cell Biol. 6, 197-208 (2005).

22. Uversky, V. N. \& Dunker, A. K. Understanding protein non-folding. Biochim. Biophys. Acta 1804, 1231-1264 (2010).

23. Babu, M. M., van der Lee, R., de Groot, N. S. \& Gsponer, J. Intrinsically disordered proteins: regulation and disease. Curr. Opin. Struct. Biol. 21, 1-9 (2011).

24. Tompa, P. Intrinsically disordered proteins: a 10-year recap. Trends Biochem. Sci. 37, 509-516 (2012).

25. Uversky, V. N., Oldfield, C. J. \& Dunker, A. K. Intrinsically disordered proteins in human diseases: introducing the D2 concept. Annu. Rev. Biophys. 37, 215-246 (2008).

26. Metallo, S. J. Intrinsically disordered proteins are potential drug targets. Curr. Opin. Chem. Biol. 14, 481-488 (2010).

27. Dyson, H. J. Expanding the proteome: disordered and alternatively-folded proteins. Q. Rev. Biophys. 44, 467-518 (2011).

28. Uversky, V. N. Intrinsically disordered proteins and novel strategies for drug discovery. Expert Opin. Drug Discov. 7, 475-488 (2012).

29. Shangary, S. \& Wang, S. Small-molecule inhibitors of the MDM2-p53 protein-protein interaction to reactivate p53 function: a novel approach for cancer therapy. Annu. Rev. Pharmacol. Toxicol. 49, 223-241 (2009).

30. Rini, J. M., Schulze-Gahmen, U. \& Wilson, I. A. Structural evidence for induced fit as a mechanism for antibody-antigen recognition. Science 255, 959-965 (1992).

31. Oldfield, C. J. et al. Flexible nets: disorder and induced fit in the associations of p53 and 14-3-3 with their partners. BMC Genomics 9(Suppl 1): S1 (2008).

32. Wilson, I. A. \& Cox, N. J. Stuctural basis of influenza virus hemagglutinin. Annu. Rev. Immunol. 8, 737-771 (1990).

33. Cheetham, J. C. et al. Antigen mobility in the combining site of an anti-peptide antibody. Proc. Natl Acad. Sci. USA 88, 7968-7972 (1991).

34. Brown, M. C. et al. Impact of immunization technology and assay application on antibody performance-a systematic comparative evaluation. PLoS One 6, e28718 (2011).

35. Chen, H. Y. et al. Monoclonal antibodies against the C(epsilon)mX domain of human membrane-bound $\operatorname{IgE}$ and their potential use for targeting IgE-expressing B cells. Int. Arch. Allergy Immunol. 128, 315-324 (2002). 
36. Otwinowski, Z. \& Minor, W. Processing of X-ray diffraction data collected in oscillation mode. Method Enzymol. 276, 307-326 (1997).

37. McCoy, A. J. et al. Phaser crystallographic software. J. Appl. Crystallogr. 40, 658-674 (2007).

38. Emsley, P., Lohkamp, B., Scott, W. G. \& Cowtan, K. Features and development of Coot. Acta Crystallogr. D Biol. Crystallogr. 66, 486-501 (2010).

39. Adams, P. D. et al. The Phenix software for automated determination of macromolecular structures. Methods 55, 94-106 (2011).

40. Murshudov, G. N. et al. REFMAC5 for the refinement of macromolecular crystal structures. Acta Crystallogr. D Biol. Crystallogr. 67, 355-367 (2011).

41. Brooks, B. R. et al. CHARMm: a program for macromolecular energy, minimization, and dynamics calculations. J. Comput. Chem. 4, 187-217 (1983).

42. MacKerell, J. A. D. et al. All-hydrogen empirical potential for molecular modeling and dynamics studies of proteins using the CHARMM22 force field. J. Phys. Chem. B. 102, 3586-3616 (1998).

43. Jorgensen, W. L., Chandrasekhar, J., Madura, J. D., Impey, R. W. \& Klein, M. L. Comparison of simple potential functions for simulating liquid water. J. Chem. Phys. 79, 926-935 (1983).

44. Darden, T., York, D. \& Pedersen, L. Particle mesh Ewald: An N.log(N) method for Ewald sums in large systems. J. Chem. Phys. 98, 10089-10092 (1993).

45. Herbert, A. MaxCluster, A tool for Protein Structure Comparison and Clustering. URL: http://www.sbg.bio.ic.ac.uk/ maxcluster/.

46. Romero, P. et al. Sequence complexity of disordered protein. Proteins 42, 38-48 (2001).

47. Shimizu, K., Hirose, S. \& Noguchi, T. POODLE-S: web application for predicting protein disorder by using physicochemical features and reduced amino acid set of a position-specific scoring matrix. Bioinformatics 23, 2337-2338 (2007)

48. Galzitskaya, O. V., Garbuzynskiy, S. O. \& Lobanov, M. Y. FoldUnfold: web server for the prediction of disordered regions in protein chain. Bioinformatics 22, 2948-2949 (2006).

49. Prilusky, J. et al. FoldIndex: a simple tool to predict whether a given protein sequence is intrinsically unfolded. Bioinformatics 21, 3435-3438 (2005).
50. Dosztányi, Z., Csizmók, V., Tompa, P. \& Simon, I. IUPred: web server for the prediction of intrinsically unstructured regions of proteins based on estimated energy content. Bioinformatics 21, 3433-3434 (2005).

51. Ishida, T. \& Kinoshita, K. PrDOS: prediction of disordered protein regions from amino acid sequence. Nucleic Acids Res. 35, W460-W464 (2007).

52. Linding, R. et al. Protein disorder prediction: implications for structural proteomics. Structure 11, 1453-1459 (2003).

53. Davis, I. W. et al. MolProbity: all-atom contacts and structure validation for proteins and nucleic acids. Nucleic Acids Res. 35, W375-W383 (2007).

\section{Acknowledgements}

This research was supported by Academia Sinica and the National Science Council in Taiwan. We thank NSRRC staff for their technical support in synchrotron data collection (Beamline BL13C1 at NSRRC in Hsinchu, Taiwan). We also thank Pheidias C. Wu for valuable discussion.

\section{Author contributions}

H.M.-C. designed and performed experiments, analysed data, wrote the first draft and prepared the figures; J.W. performed molecular dynamics simulations; Y.-H.C. performed ELISA experiments; C.-J.L. prepared IgG1.Fc-CemX-migis fusion protein, T.W.C. and C.L. conceived and supervised the project, analysed the data and edited the paper and figures.

\section{Additional information}

Accession code. The atomic coordinates reported in this paper have been deposited in Protein Data Bank with accession code 4LKX

Competing financial interests: The authors declare no competing financial interests.

Reprints and permission information is available online at http://npg.nature.com/ reprintsandpermissions/

How to cite this article: Chu, H.-M. et al. Two potential therapeutic antibodies bind to a peptide segment of membrane-bound IgE in different conformations. Nat. Commun. 5:3139 doi: 10.1038/ncomms4139 (2014). 